\title{
Diseño e Implementación de un Sistema de Control y Monitoreo Remoto para la Iluminación de una Vivienda a través de un Teléfono Móvil con Sistema Operativo Android y Board ARM mini2440
}

\section{Design and implementation of a Remote Lighting Control and Monitoring System Using Android and Board ARM mini2440 Mobile Operating Systems}

Jesús David Quintero P. ${ }^{[1]}$, Eliana Fernanda Tacan C. ${ }^{[2]}$ y Fernando Augusto España C. ${ }^{[3]}$

\begin{abstract}
Resumen
El presente proyecto se realizó con el fin de lograr el control y monitoreo remoto del sistema de luces de una vivienda, construida a escala (1:10), que funciona con la red eléctrica (110V), a través de un teléfono móvil que cuente con sistema operativo Android. Se adecuó una etapa de potencia para cada bombillo, la cual ajusta la señal digital a la red eléctrica. Se creó una aplicación web móvil utilizando lenguaje de programación HTML5, dando la posibilidad de que dicha aplicación sea vista desde diferentes dispositivos móviles a través de un navegador web o desde una aplicación en un teléfono móvil con sistema operativo Android. Dicha aplicación posee una interfaz gráfica sencilla, en la que se muestra el nombre del lugar donde se encuentra cada bombillo, además, cuenta con una base de datos hecha en SQLite3, la cual, enseña el tiempo que éste lleva encendido y da la opción de introducir la potencia de cada bombillo, dando la posibilidad de conocer el valor, en pesos, que el usuario debe pagar por la energía consumida de cada bombillo; esto se hizo ya que las especificaciones de cada bombillo son diferentes.
\end{abstract}

Palabras clave: ARM mini2440; control de luces; monitoreo remoto; Android; SQLite3; HTML5; aplicación web.

\begin{abstract}
The purpose of this project was to design and implement a lighting control and monitoring system for housing, the system was built to a scale of (1:10), and works with an (110V) electrical network. It is operated through a cellular phone with Android operating system. A power amplifier was adapted for each light buld, which adjusts the digital signal to the electrical network. We created a mobile Web application using HTML5 programming language making it possible for the application to be accessed from different mobile
\end{abstract}

[1] Magister docente. Universidad Surcolombiana, Neiva. Avenida Pastrana Carrera 1ª. jdavid@usco.edu.co

[2] Ingeniero Electrónico. Universidad Surcolombiana, Neiva. Avenida Pastrana Carrera 1ª. fernandat2130@gmail.com

[3] Ingeniero Electrónico. Universidad Surcolombiana, Neiva. Avenida Pastrana Carrera 1ª faespa.88 @gmail.com

Recibido: 11 abril 2015 • Aceptado: 3 mayo 2015. 
devices through either a navigator or a mobile application for Android. This application has a simple graphic interface, which shows where the light bulb is located, as well as an SQLite3 database, which shows how long the light bulb has been on for. This also provides an option introduce an the power output of each light bulb, making it possible for the user to know the value, in pesos, to be paid for the electricity used. This was undertaken because the specifications of each light bulb are different.

Key words: ARM mini2440, lighting control, remote control, Android; SQLite3; HTML5; Web application.

\section{Introducción}

En un mundo como el de hoy en día, en donde la ciencia y la tecnología avanzan a pasos agigantados, el ser humano debe proveerse y estar atento a estos cambios, los cuales, en su gran mayoría, se hacen para su bienestar y beneficio (Betancourt, 2009).

Hace poco más de 50 años, el ser humano se encontraba en un proceso de creación tecnológica sin precedentes. Transistores y microcontroladores fueron la base de la electrónica moderna, haciéndolos cada vez más pequeños pero con mayor capacidad y velocidad. Con la creación de los computadores, se dio un gran salto tecnológico hacia lo moderno. Tarjetas perforadas ahora podían ser interpretadas dentro de una interfaz gráfica la cual era visible por el ser humano. Se pasó de las máquinas de escribir a tener un entorno visual en donde el hombre y la máquina podían interactuar de una manera rápida y sencilla, permitiendo procesar y almacenar información sin temor a que ésta se perdiera. Se siguió avanzando, y todo era cada vez más pequeño, pero a la vez mejor que su antecesor. De los grandes computadores de mesa se pasaron a los computadores portátiles, pequeños aparatos electrónicos, capaces de cumplir con las funciones de un computador de mesa, pero con la diferencia de que se podía llevar a donde se quisiera.

Tener una rápida, sencilla y estable comunicación con un ser querido que estaba lejos era toda una odisea. Las cartas se podían perder en el camino y el uso de internet era muy reducido, casi que solo las grandes multinacionales podían acceder a éste. Gracias a ésta y muchas otras necesidades, nacen los teléfonos móviles, pequeños dispositivos capaces de comunicarse vocal e inalámbricamente con cualquier persona en el mundo sin importar donde ésta se encuentre.

La domótica o casas inteligentes, es una prueba de lo mucho que puede aportar la tecnología al bienestar del hombre. Desde el encendido y apagado de las luces, pasando por la apertura y cierre de puertas, hasta el control de la cocina, nevera, etc., la domótica se ha convertido hoy en día en una herramienta muy útil a la hora de interactuar con los objetos que componen una casa. Todo esto puede ser logrado, ya sea desde un computador de mesa o computador portátil, incluso desde un Smartphone, gracias a la fácil interacción que tiene el ser humano con la tecnología (Casadomo, 2003).

En un mundo donde la innovación está a la vuelta de la esquina, no es raro que las personas vivan atrasadas de los adelantos tecnológicos, sin embargo, cada día se hace más sencillo lograr acceder a este tipo de información. Los teléfonos inteligentes logran tener aplicaciones web de todo tipo, desde noticias hasta juegos, pasando por las redes sociales y llegando hasta el control total de una residencia. A través de este tipo de dispositivo, es posible lograr el control de todas las cosas electrónicas que se encuentren dentro, dando a los usuarios la tranquilidad, confort y bienestar que se merecen (Herrera, 2012).

Este proyecto está basado en un sistema capaz de controlar y monitorear las luces de una vivienda por medio de un teléfono móvil a través de un sistema embebido (Llinares, 2014) el cual es el encargado, gracias a su programación, de interconectar las luces del prototipo de vivienda con el teléfono. Dicho control y monitoreo varía de acuerdo a las especificaciones de la vivienda, por consiguiente, esta aplicación es compatible únicamente con la maqueta en la que se prueba el sistema.

Ya que hay diferentes formas de lograr la comunicación entre el sistema embebido y el dispositivo móvil, se realizó un estudio, de tal forma que la persona que use la aplicación pueda encender o apagar las luces desde cualquier lugar, siempre y cuando el dispositivo móvil esté en red con el servidor de la board (Donelson, 2008). A través de una aplicación web instalada en el teléfono móvil, la persona podrá controlar el encendido y apagado de las luces de la vivienda, y por medio de la base de datos, podrá monitorear el consumo de 
cada una, logrando así un ahorro económico (Mateu, 2004).

\section{Metodología}

El sistema de control y monitoreo remoto de los bombillos se realizó mediante el uso del puerto GPIO (ARMWorks, 2009) de la board ARM mini2440, la cual, utiliza un circuito de potencia para acoplar los elementos a la red eléctrica. Esta tarjeta hará las veces de servidor, y será a través de ella que se logre enviar y recibir las peticiones del cliente. En el puerto GPIO se seleccionaron 5 pines como entrada y 5 pines como salida. En los pines de entrada se conectaron los pulsadores y en los de salida los bombillos, los cuales van antecedidos por el circuito de potencia. Se hace uso de un nano Router de marca TP-Link referencia TL-WR702N para que todo el circuito esté en la misma red, permitiendo la interacción del sistema con el usuario.

Dado que es un sistema pensado para controlar las luces de una casa remotamente (hogar domótico), se cambian los tradicionales interruptores por pulsadores, simulando interruptores digitales, los cuales al pulsarse, permitan al usuario encender o apagar el bombillo manualmente. Todos los datos serán registrados en una base de datos hecha en SQLite3 (Aguiar, 2006), en dónde se podrá observar la hora y la fecha de encendido y apagado de los bombillos, mostrar el bombillo que se encendió y el tiempo que éste lleva encendido. Esta base de datos estará alojada en el servidor.

Para el diseño de control se trabajó desde un computador de escritorio que contara con sistema operativo Linux (en este caso se utilizó la distribución OpenSuse en su versión 12.3), ya que la compilación del código sólo se puede hacer a través de la consola de este sistema.

La aplicación web se encargará de la interfaz gráfica para que el usuario pueda interactuar cómodamente con el control de los bombillos, ya sea accediendo a la aplicación desde un teléfono móvil con tecnología Android o desde cualquier dispositivo móvil con navegador web ingresando la dirección IP del servidor (192.168.1.230). Desde ahí se podrá observar el tiempo real que lleva encendido cada bombillo, y, al introducir la potencia, en wattios, el usuario podrá saber cuánto dinero debe pagar hasta ese momento por el consumo de los bombillos. Se crearon dos botones: uno para eliminar la tabla de registros, pero esto sólo elimi- naría la tabla, ya que los registros siguen almacenados, si no, las consultas no se podrían realizar, y otro, para ver los registros que están siendo almacenados en la base de datos. Al haber varios dispositivos conectados al servidor, se puede ver el cambio casi que instantáneo (ya que tiene un pequeño retardo) del estado de los bombillos, además, el tiempo que éstos llevan encendidos sólo será sincronizado cuando el bombillo respectivo se apague. El esquema general del sistema se puede observar en la Figura 1.

\subsection{Hardware}

El centro de control del sistema es la board ARM mini2440. En este sistema embebido estará alojado todo el código para el control y monitoreo del sistema de luces de la vivienda construida a escala (1:10). Este sistema embebido contará en su periferia con componentes electrónicos los cuales son requeridos para la conexión y el correcto funcionamiento del sistema.

\subsubsection{Board ARM mini2440}

Es un dispositivo de origen Chino, el cual es distribuido, a nivel internacional, por compañías como FriendlyARM e Industrial ARMWorks. Se podría decir que se trata de un sistema de computación versátil y de propósito múltiple, pero con notables limitaciones de recursos con respecto a un PC.

Sorprende la arquitectura con la que viene la tarjeta, pues para ser un componente de baja potencia, trabaja con tecnología RISC (ARM920T de 32 bits), siendo los ciclos de reloj mucho más rápidos que su oponente CISC. Utiliza un micro-controlador fabricado por la empresa surcoreana Samsung (S3C2440A), CMOS de 32 bits. Tiene dos memorias SDRAM de $32 \mathrm{Mb}$ (64 Mb total), una memoria NAND Flash (o almacenamiento) de $1024 \mathrm{Mb}$, una memoria NOR Flash de 2 $\mathrm{Mb}$ y un chip de red Ethernet. Cuenta con una serie de interfaces (ver Figura 2) las cuales permiten realizar diferentes tareas. Dentro de las más destacadas tenemos: puerto GPIO de 34 pines, conector RJ-45, conector USB esclavo tipo B hembra y una interfaz de alimentación de 5V (ARMWorks, 2009).

$\mathrm{Al}$ ser una tarjeta de desarrollo, son múltiples las tareas que programadores o desarrolladores pueden hacer, teniendo la posibilidad de probar ahí mismo sus aplicaciones, además, su robustez y su precio reducido hacen que esta tarjeta sea de gran utilidad en el campo de la docencia y la investigación (Zablaza, 2011). 


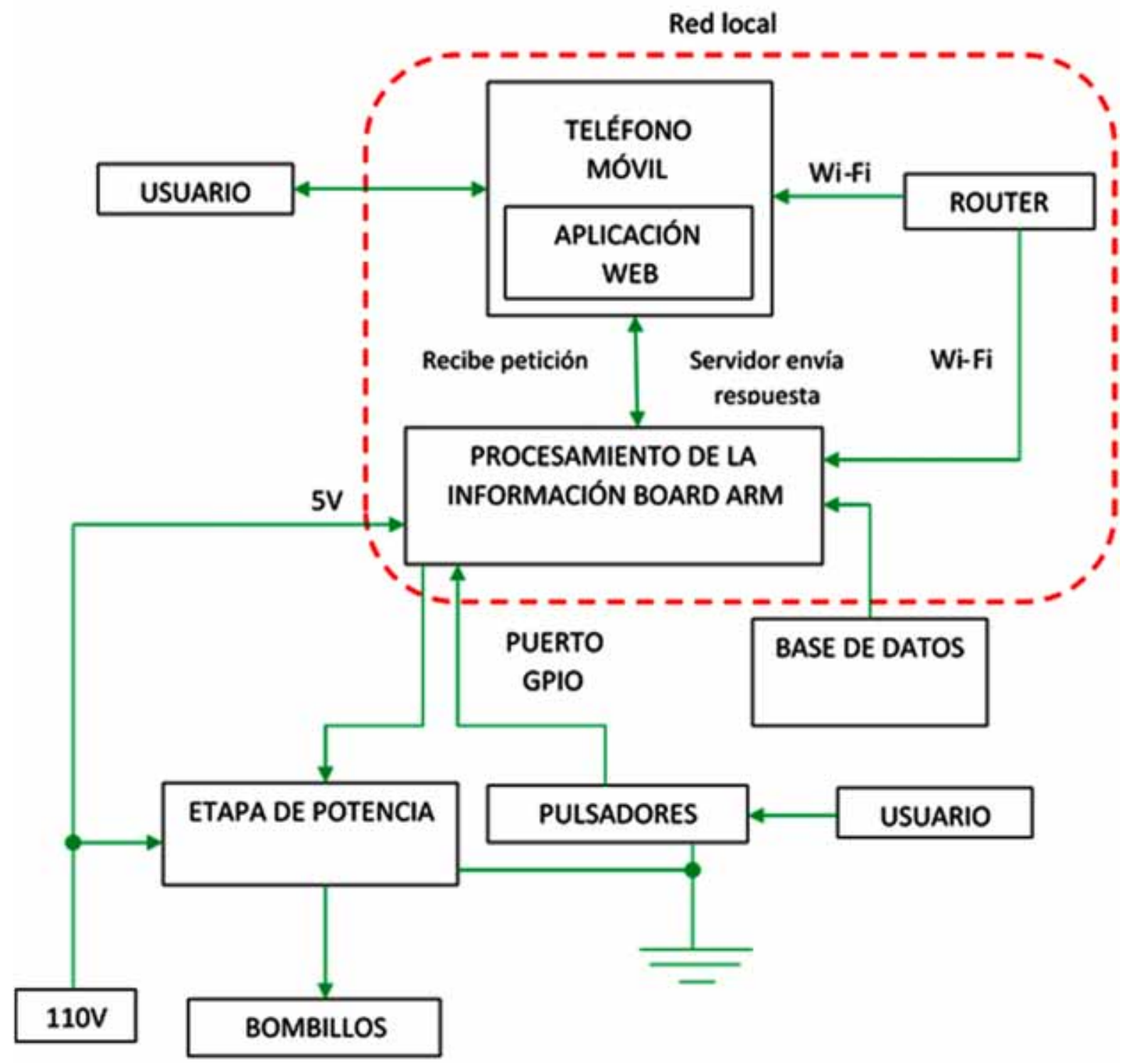

Figura 1. Esquema general del sistema

La tarjeta está construida sobre una tarjeta de 10 x 10 $\mathrm{cm}$, y cuenta con una gran variedad de interfaces y sistemas de almacenamiento, los cuales, están montados sobre la placa. Incluye una pantalla LCD de 3.5”, la cual está sobre la tarjeta y es sujetada a través de tornillos, en caso de que el usuario quiera quitarla para, ya sea ver sus referencias o trabajar, no tenga ningún inconveniente y su interacción sea mucho más sencilla. El PCB (tarjeta de circuito impreso) de la tarjeta está diseñado para ser de 4 capas, adoptando tecnología ENIG y cableado profesional de igual longitud para asegurar la integridad de las señales del cable de señal clave.

Los pines del puerto GPIO pueden ser usados tanto de entrada como de salida. Para este caso se definió unos pines de salida (para encender y apagar los bombillos a través del dispositivo móvil) y otros de entrada (para encender y apagar los bombillos a través del pulsador).

Para ser usado como salida se definió los siguientes pines: CON4_PIN9, CON4_PIN13, CON4_PIN17, CON4_PIN21, CON4_PIN25.

Para ser usado como entrada se definió los siguientes pines: CON4_PIN12, CON4_PIN16, CON4_PIN20, CON4_PIN24, CON4_PIN28.

Se dispuso así, de forma intercalada, dado que no se consiguió un bus para acoplar los pines del puerto GPIO. En vez de eso, a cada pin se le adecuó un pin 


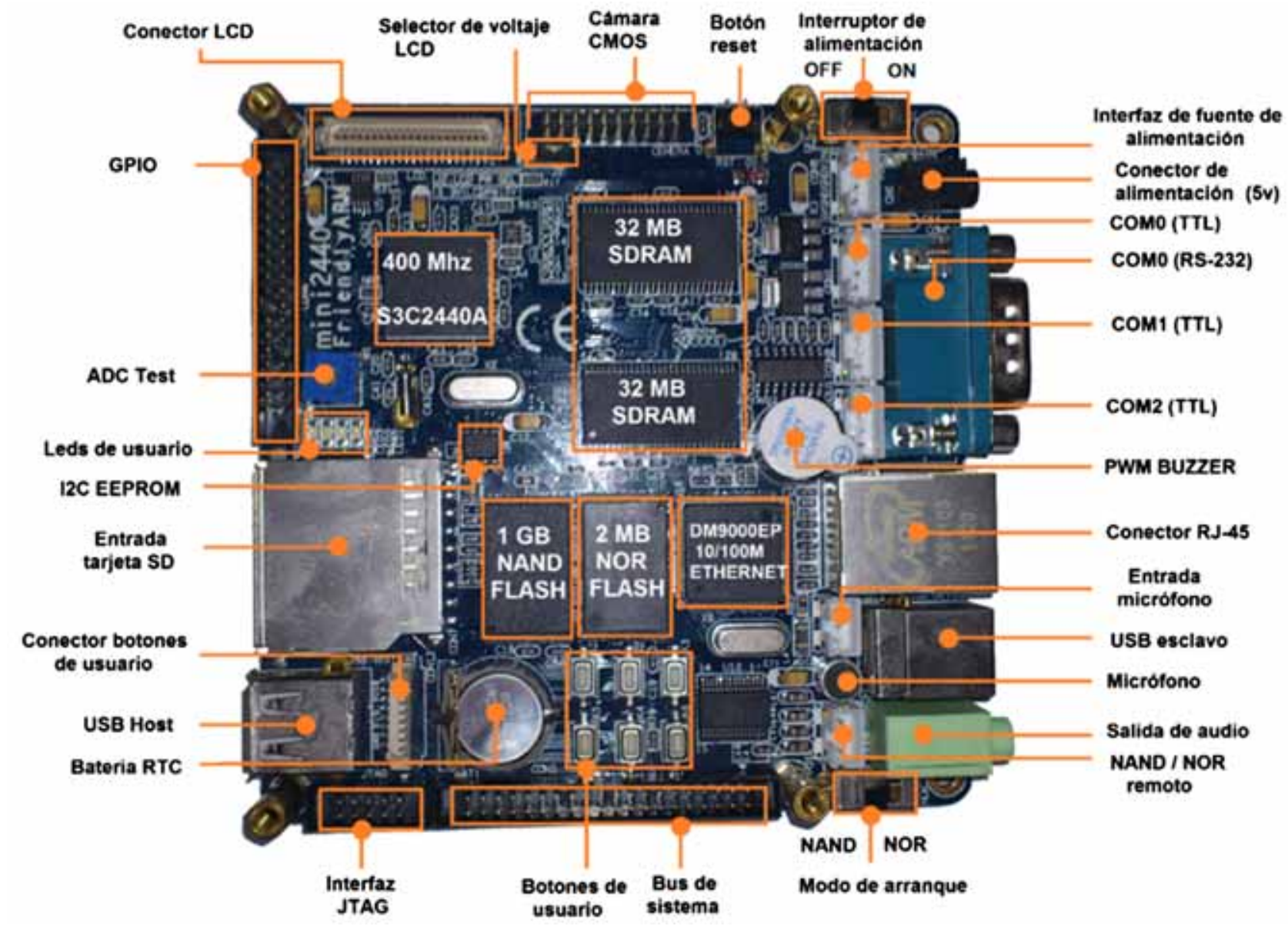

Figura 2. Board ARM mini2440,

de una regleta para lograr tener un poco más largo los pines del puerto GPIO. El pin 3 será la tierra común para los pulsadores.

\subsubsection{Circuito de acople}

Dado que el sistema embebido es un elemento de baja potencia, se diseñó un circuito de acople, de tal forma que los circuitos que se vayan a integrar con dicha tarjeta no sufran daño alguno, y menos la propia tarjeta de desarrollo. El circuito de acople que se construyó fue una etapa de potencia para acoplar la señal digital a la red eléctrica, debido a que con la tarjeta solo se consiguen sacar 5V, los cuales no son suficientes para encender un bombillo que trabaja con $110 \mathrm{~V}$. Se realizó una (1) etapa de potencia para cada bombillo (ver Figura 3).

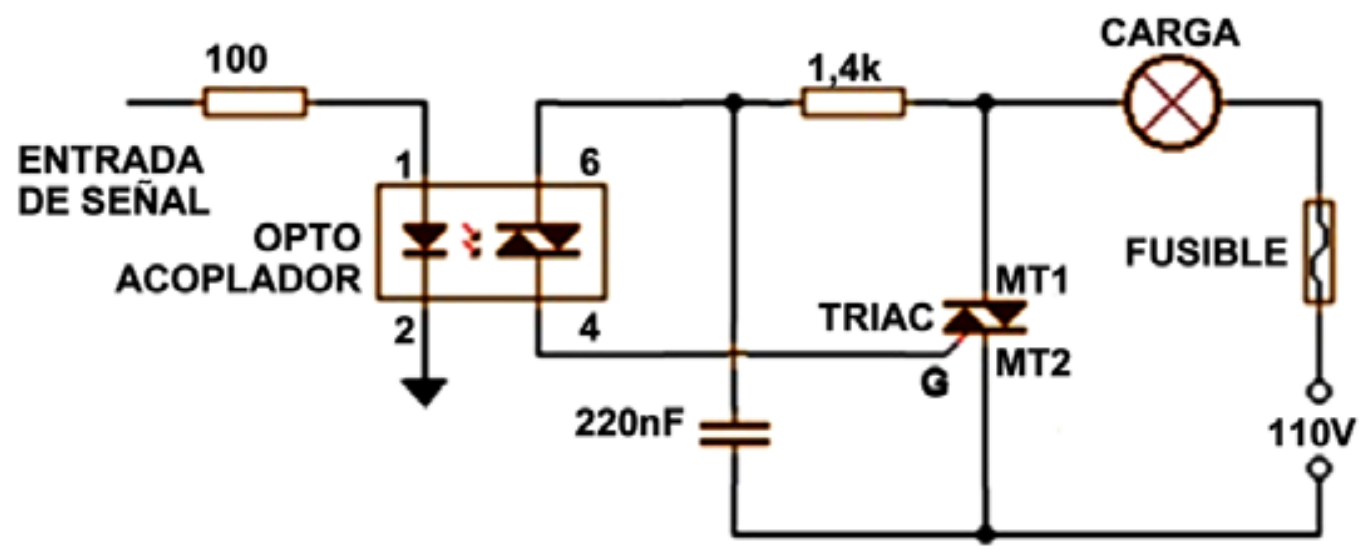

Figura 3. Circuito esquemático de control de potencia para los bombillos. 


\subsubsection{Nano router TP-Link TL-WR702N}

Debido a que se debe contar con una conexión inalámbrica entre la tarjeta y el dispositivo móvil, es necesario crear una red local. Para realizar la red se utilizó un nano router inalámbrico de la familia TPLink de referencia TL-WR702N. Se eligió este router dada su facilidad de uso. Puede alcanzar velocidades de datos inalámbricos de hasta 150Mbps y su conexión a la energía se puede dar ya sea por conexión USB hasta un ordenador o por medio de un adaptador de energía externo (ver Figura 4).

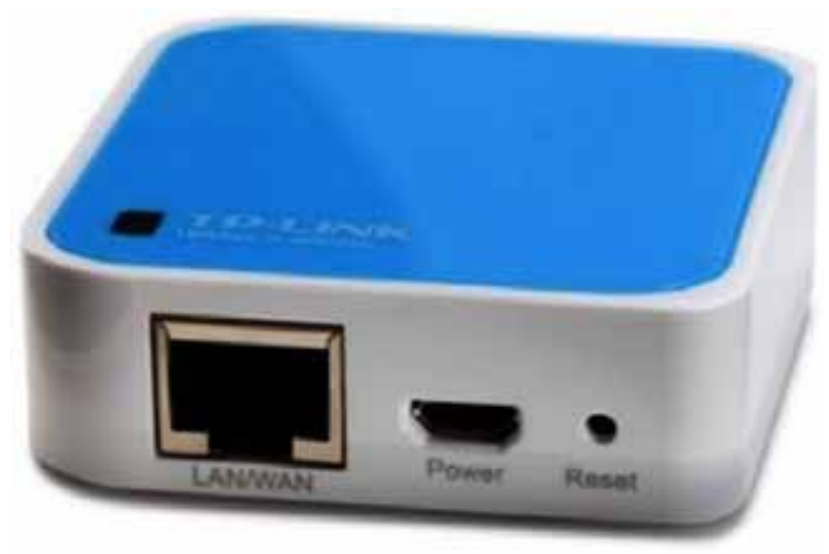

Figura 4. Nano router TP-Link TL-ER702N.

\subsection{Software}

Dada la complejidad de la configuración del hardware de esta tarjeta, está diseñada para ser dominada por un sistema operativo que gestione los procesos básicos del sistema. El sistema tiene suficiente capacidad de procesamiento y memoria para albergar y ejecutar un sistema operativo multitarea/multiusuario con una interfaz gráfica de usuario completa.

Esto significa que la persona que utilice esta tarjeta de desarrollo deberá primero instalar un sistema operativo previamente compilado para esta arquitectura, junto con los controladores de los subsistemas. Luego de esto, podrá programar y desarrollar sus aplicaciones haciendo uso de las plataformas y librerías disponibles que le puedan dar soporte.

Al día de hoy, existen varias opciones de sistemas operativos para la ARM mini2440, dentro de los cuales se encuentran: Linux, Android 3.0 y Windows CE.

Para el desarrollo del presente proyecto se escogió por sistema operativo una distribución de Linux llamada Qtopia (ver Figura 5) en su versión 2.2.0 (hoy en

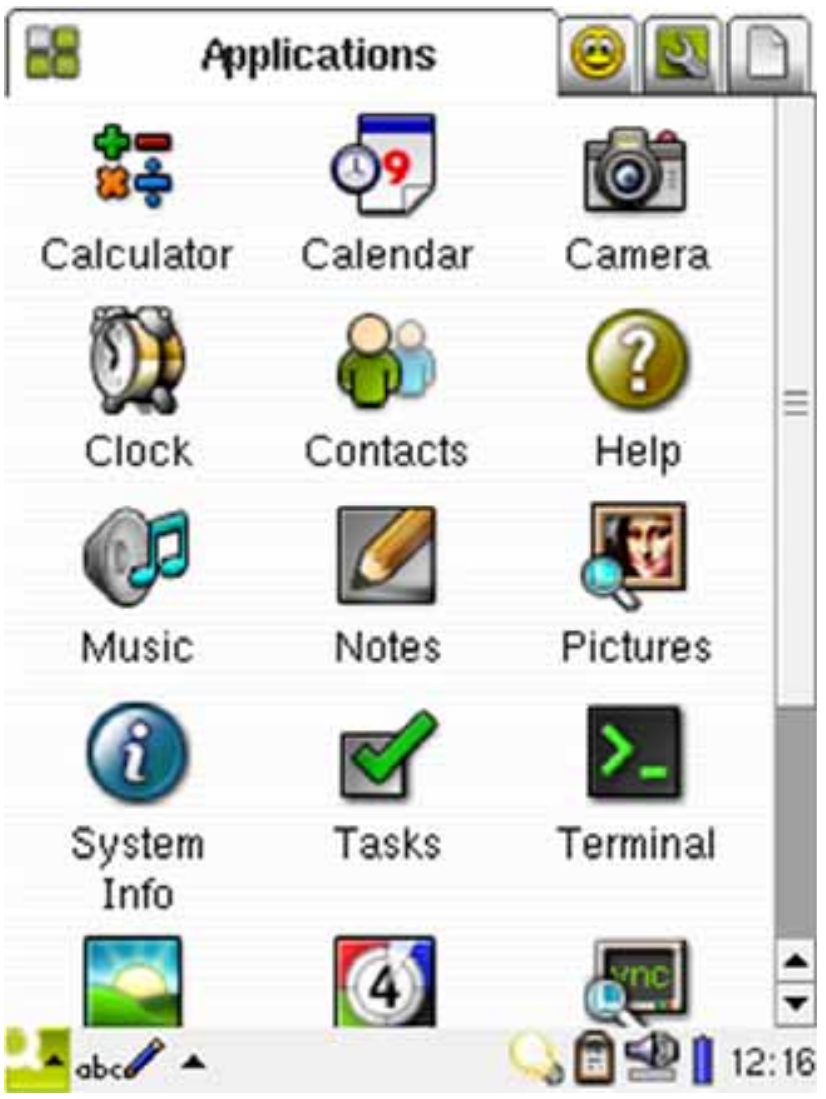

Figura 5. Entorno gráfico Qtopia 2.2.0

día se conoce como Qt Extended), ya que se sabe que Linux es un sistema operativo muy potente, seguro y gratuito. Este sistema operativo es una plataforma de aplicaciones para dispositivos móviles y está desarrollado bajo la licencia GPL (Opie).

\subsection{Comunicación Board ARM mini2440 - PC}

Luego de cambiar el sistema operativo por defecto que trae la board ARM mini2440, se estableció una comunicación entre ésta y el PC, para mayor comodidad de trabajo. Por defecto, la tarjeta se puede trabajar desde la consola que se encuentra ubicada dentro de la board, pero este trabajo es muy tedioso, ya que, al no tener teclado físico, el usuario debe escribir, por medio del lápiz óptico, letra por letra en la consola, haciendo el trabajo más largo y dispendioso, o también, se puede trabajar de forma remota gracias al protocolo de red que trae por defecto llamado TELNET. Este protocolo de red permite la comunicación remota con la tarjeta pero no cifra los archivos, dejando residuos en la red al alcance de terceros; además, la transferencia de archivos sólo se podía realizar a través del protocolo FTP, impidiendo la transferencia de archivos completa. 
Dado que el protocolo de red multiplataforma TELNET no cumplió con las expectativas esperadas, se decidió utilizar otro protocolo de red el cual fuera compatible con la tarjeta y permitiera la transmisión y recepción de datos completa y segura. Para esto, se utilizó el protocolo de red OpenSSH (Open Secure Shell, Abrir intérprete de órdenes segura), el cual es soportado por la tarjeta.

La board ARM mini2440 cuenta con una dirección IP la cual se utiliza para acceder a ésta y funciona como servidor. La dirección para tener acceso es 192.168.1.230. Se debe aclarar que para lograr tener acceso se debe estar en red con el servidor de la tarjeta. A través de esta dirección IP se puede acceder de forma remota a la tarjeta desde un terminal.

Para llevar a cabo la transferencia de archivos se utilizó un programa llamado FileZilla. Por medio de éste es posible llevar a cabo una conexión con la máquina a través de diferentes protocolos (FTP, SFTP, FTPS, FTPES). Este cliente multiplataforma sirve para ser usado tanto en versiones de Windows como en versiones de Linux.

\subsection{Herramientas de desarrollo empleadas}

Lograda la comunicación remota, se utilizó algunas herramientas las cuales eran primordiales para el desarrollo y culminación del proyecto.

\subsubsection{Sistema operativo PC: OpenSUSE}

Dado que la tarjeta solo se puede compilar desde un sistema operativo con Linux, en este caso se utilizó la distribución OpenSUSE en su versión 12.3. Se eligió este $\mathrm{SO}$ ya que su interfaz gráfica tiene bastante similitud con Windows, haciendo su manejo mucho más sencillo, además de que tiene la opción de que el usuario pueda elegir entre dos (2) entornos de escritorio: Gnome o KDE. Es gratuito, pues se puede descargar desde su página oficial en internet, totalmente estable y de fácil instalación (se graba la imagen obtenida en un CD dejándolo auto-arrancable y siguiendo los pasos de instalación), además, cuenta con una amplía comunidad de usuarios los cuales brindan soporte.

\subsubsection{Compilador cruzado ARM LINUX GCC 4.3.2}

Normalmente la board no tiene la misma arquitectura que una computadora de propósito general. El código fuente debe ser compilado para ser ejecutado en una plataforma distinta a aquella en la que éste se ejecuta.
Debido a que en los sistemas embebidos es imposible compilar archivos (dada la limitación de recursos), se utilizó un compilador cruzado.

Para este caso se utilizó el compilador cruzado ARM LINUX GCC 4.3.2, debido a que es el único que soporta la tarjeta embebida. Existen varias versiones de este compilador cruzado, pero la versión 4.3.2 fue la única soportada por la board ARM mini2440, las otras versiones presentaron fallos a la hora de la compilación. Este compilador recibe un programa fuente de cualquier de los lenguajes de programación anteriormente mencionados y genera un programa ejecutable binario en el lenguaje de máquina donde ha de correr, para este caso genera un archivo ejecutable con extensión CGI, permitiendo la comunicación entre el sistema embebido y la aplicación externa.

\subsubsection{Base de datos SQLite3}

La base de datos SQL soportada por la tarjeta es la SQLite3. Este es un sistema de gestión de bases de datos relacionales. Es de dominio público, escrito en lenguaje $\mathrm{C}$ y muy ligero. Soporta diferentes plataformas como Windows, Linux y Mac OS. Este tipo de base de datos se le puede llamar empotrada, ya que no inician un servicio en una máquina independientemente de la aplicación, pudiéndose enlazar directamente al código fuente o bien utilizarse en forma de librería (\#include), que es la manera como se utilizó en este caso.

Si se quiere usar la base de datos SQLite para la board ARM en aplicaciones con lenguaje $\mathrm{C} / \mathrm{C}++$ se debe incluir la librería en la cabecera del proyecto (\#include sqlite3.h) y en la línea de comandos, cuando se haga la compilación del archivo, se debe agregar la opción -l sqlite3. Esta instalación se debe realizar desde un sistema operativo Linux.

\subsection{Diseño del programa}

Debido a que se desea controlar los bombillos de una casa y al mismo tiempo llevar los registros de encendido y apagado de éstos en una base de datos, se realizó código por separado, generando archivos para cada una de las funciones.

La función gpiosfinal.cgi consulta el estado de cada bombillo y recibe las ordenes que el usuario desea realizar desde el celular, si desea prender o apagar un bombillo este verifica el estado y realiza la acción correspondiente guardando el estado de cada bombillo, esta función también se encarga de mostrar la tabla 
que contiene la base de datos de la aplicación y de eliminar la misma. El programa gpiosfinal.cgi es el encargado de manejar las acciones que realiza el usuario con la aplicación desde el celular.

La función start.cgi se encarga de iniciar la aplicación. Esta función siempre está en ejecución, además, recibe las pulsaciones que realiza el usuario. Verifica en qué estado se encuentran los bombillos y realiza la acción correspondiente, seguidamente, guarda el estado de los bombillos y los registra en una tabla. Dicha tabla será la base de datos almacenada en el servidor. Este programa, start.cgi, se ejecuta desde el momento en que se inicia la tarjeta y es el encargado de iniciar la aplicación. Si este archivo no es ejecutado, el sistema no funcionará.

Para entender mejor el funcionamiento de los programas se creó un diagrama de arquitectura (ver Figura 6).

\subsection{Aplicación web}

Las aplicaciones web son aplicaciones que corren sobre un navegador web, en este caso, sobre el navegador web del dispositivo móvil.
Para que el usuario visualice los bombillos que se encienden y apagan remotamente, además de la base de datos, se creó una aplicación web con lenguaje HTML5, la cual cuenta con una interfaz gráfica sencilla y de fácil uso. Dicha aplicación web se creó a partir del framework de LungoJS. Este framework proporciona las herramientas necesarias para la creación de páginas web para que sean visibles desde los diferentes dispositivos móviles (teléfonos, tabletas y PC).

Dado que se debe visualizar en un teléfono móvil con sistema operativo Android, fue necesario utilizar el entorno de desarrollo Eclipse para acoplar la aplicación y generar una aplicación ejecutable para Android. Para Lograr esto, el SDK de Android proporciona una clase denominada WebView para acoplar páginas web (ver Figura 7), ya sea obteniéndolas a través de una URL o bien recibiendo el html directamente desde una Activity.

\section{Resultados}

Recopilando todo lo anteriormente mencionado, se consiguió construir un sistema capaz de controlar y monitorear remotamente el sistema de luces de una

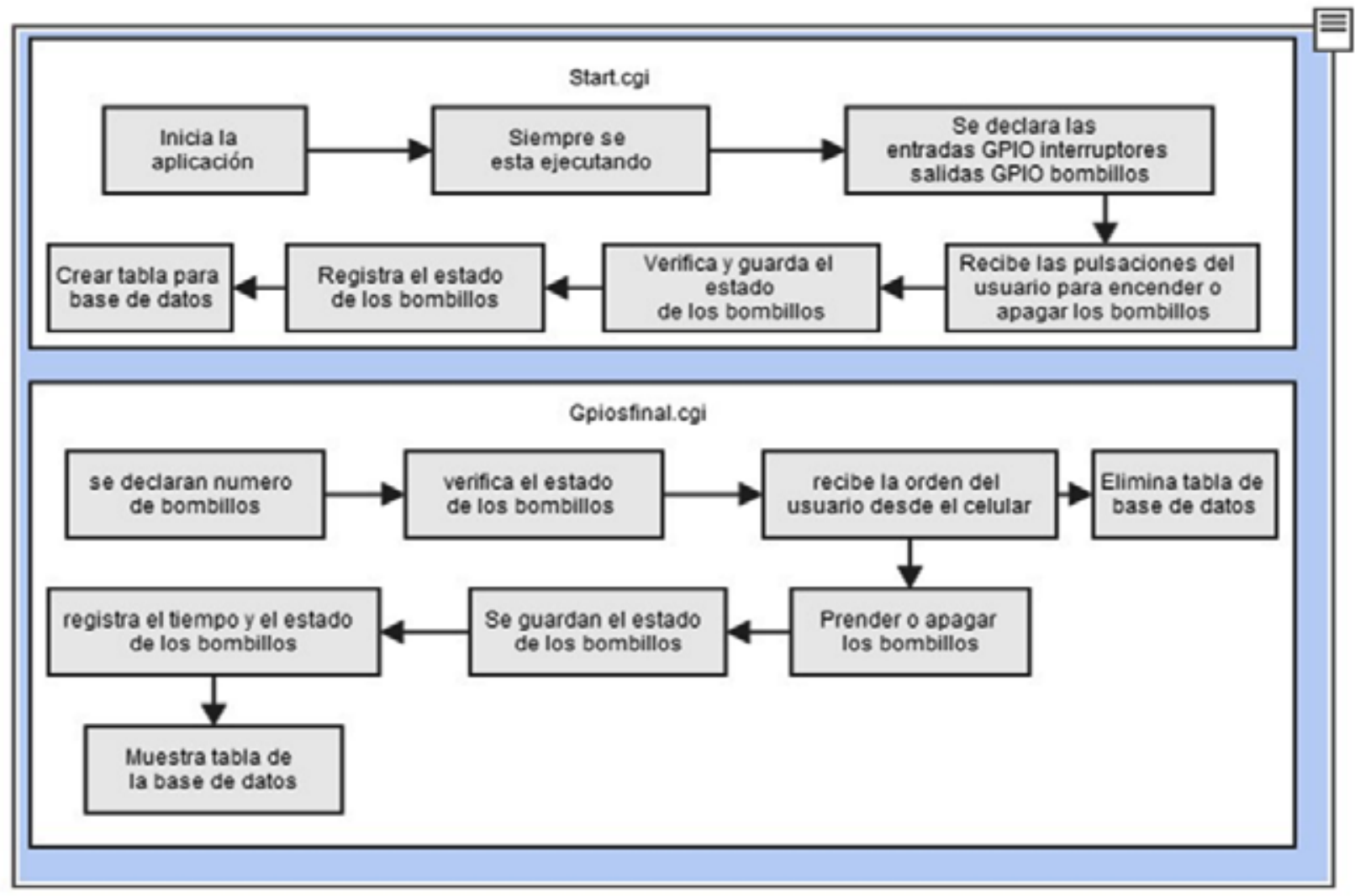

Figura. 6. Diagrama de arquitectura de desarrollo del sistema. 

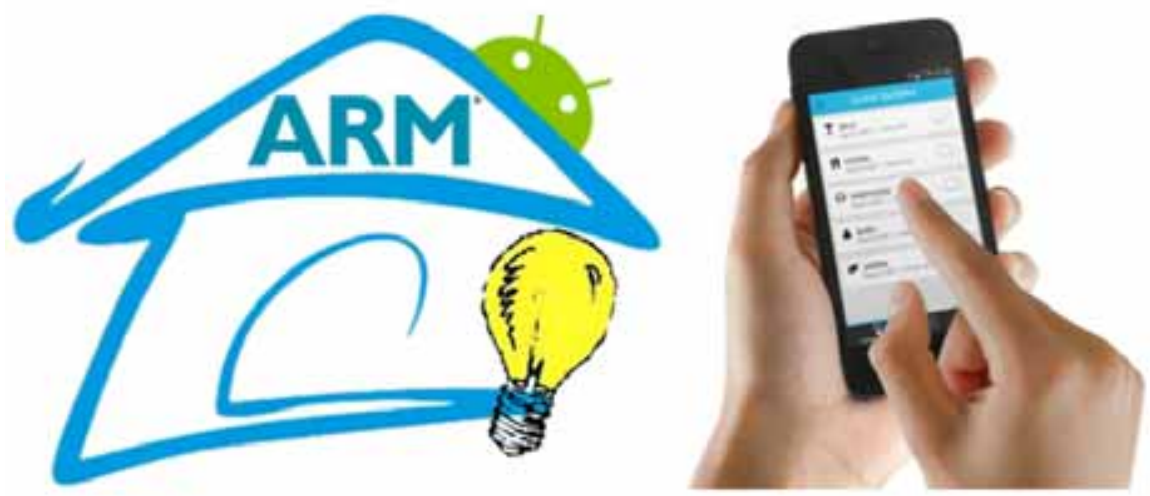

Figura. 7. Logo aplicación en dispositivo con sistema operativo Android

vivienda, a través de la board ARM mini2440, y cuya interfaz gráfica puede ser vista desde cualquier dispositivo móvil que cuente con un navegador web (ver Figura 8).

El usuario puede tener un control absoluto sobre los bombillos, pudiendo encenderlos o apagarlos ya sea de forma manual o remotamente, de todos modos, de cualquiera de las dos formas el cambio será registrado en la base de datos y podrá consultarla cada vez que quiera hacerlo. Además, la aplicación web cuenta con un botón de reset, el cual permite al usuario eliminar la tabla de la base de datos y empezar nuevamente a tomar el control del consumo de energía.

La aplicación web consta de tres (3) opciones de menú: Control Bombillos, el cual muestra el lugar dónde se llevará a cabo el control de encendido/apagado de los bombillos, haciendo dicho control a través de un botón deslizable de derecha a izquierda y

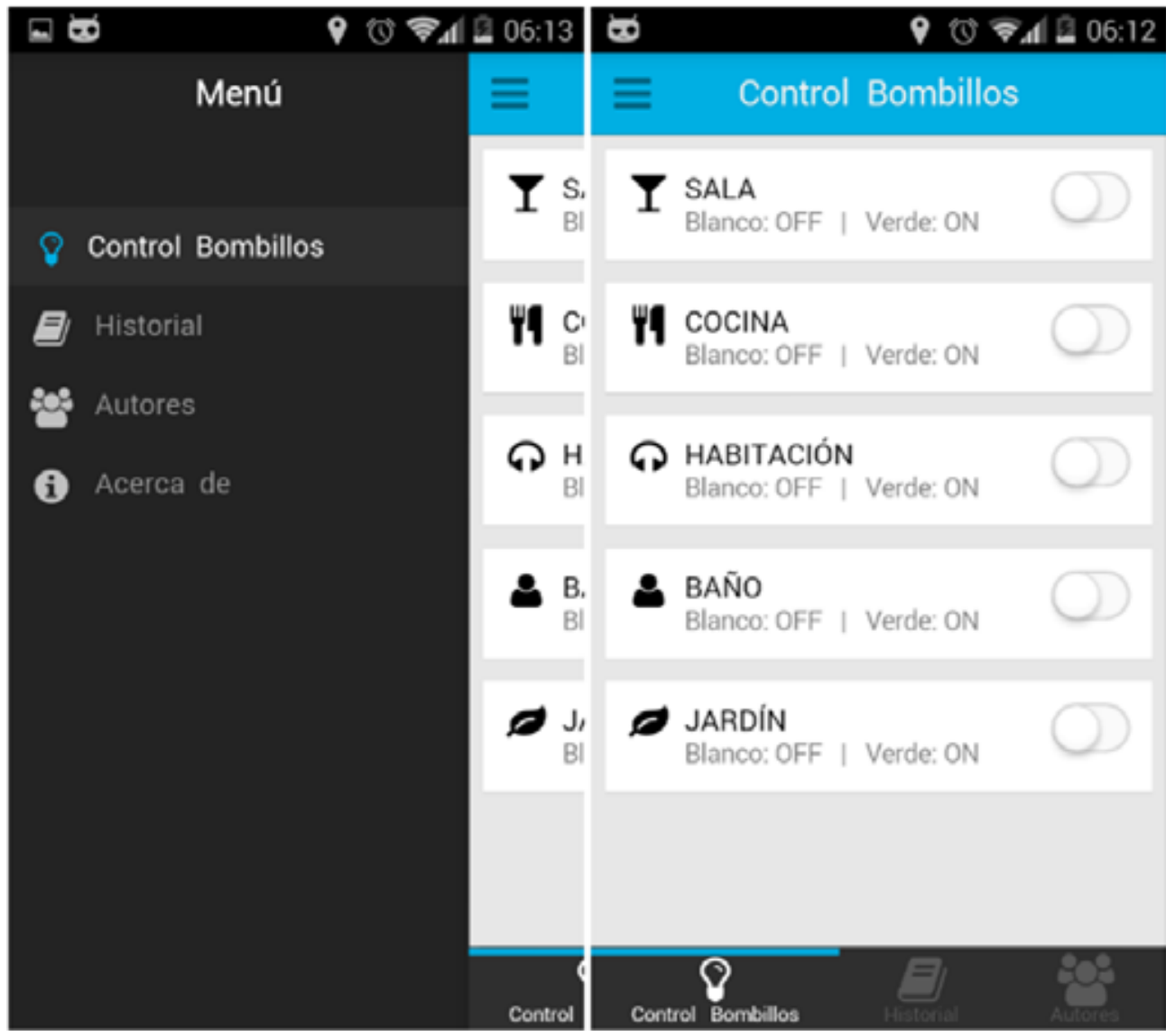

Figura 8. Interfaz gráfica aplicación web 
viceversa; Historial (ver Figura 9), el cual es dónde se muestra el tiempo real que lleva encendido cada bombillo, da la opción de introducir la potencia del bombillo para arrojar el precio que se debe pagar hasta el momento por el consumo de ese bombillo y aloja dos (2) botones para ver o eliminar la tabla de la base de datos; y Autores, que es donde se muestra la información de los desarrolladores del proyecto, incluyendo su página oficial a las redes sociales como Facebook y Twitter.

Se construyó una caja metálica (ver Figura 10) en donde se introdujo la board ARM mini2440 y la etapa de
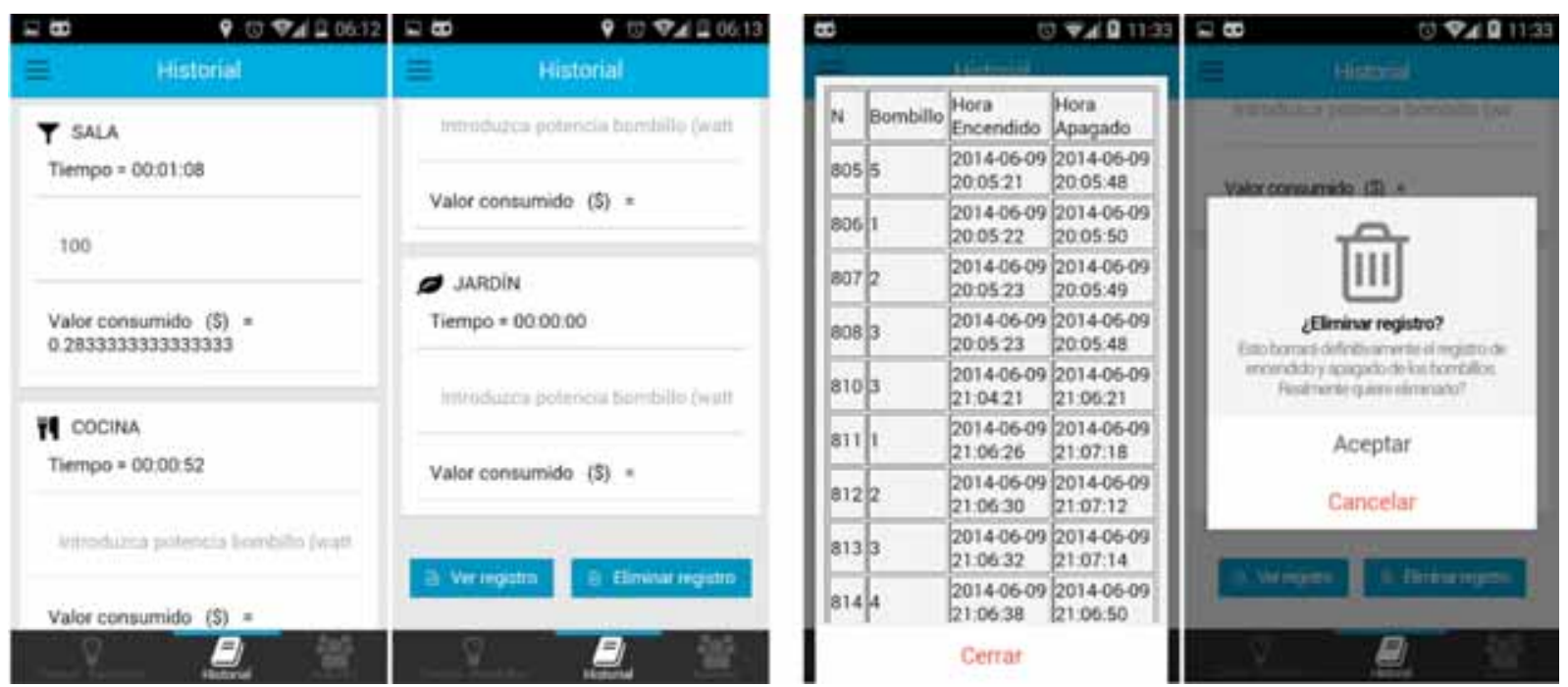

Figura 9. Aplicación web: Historial y registros.
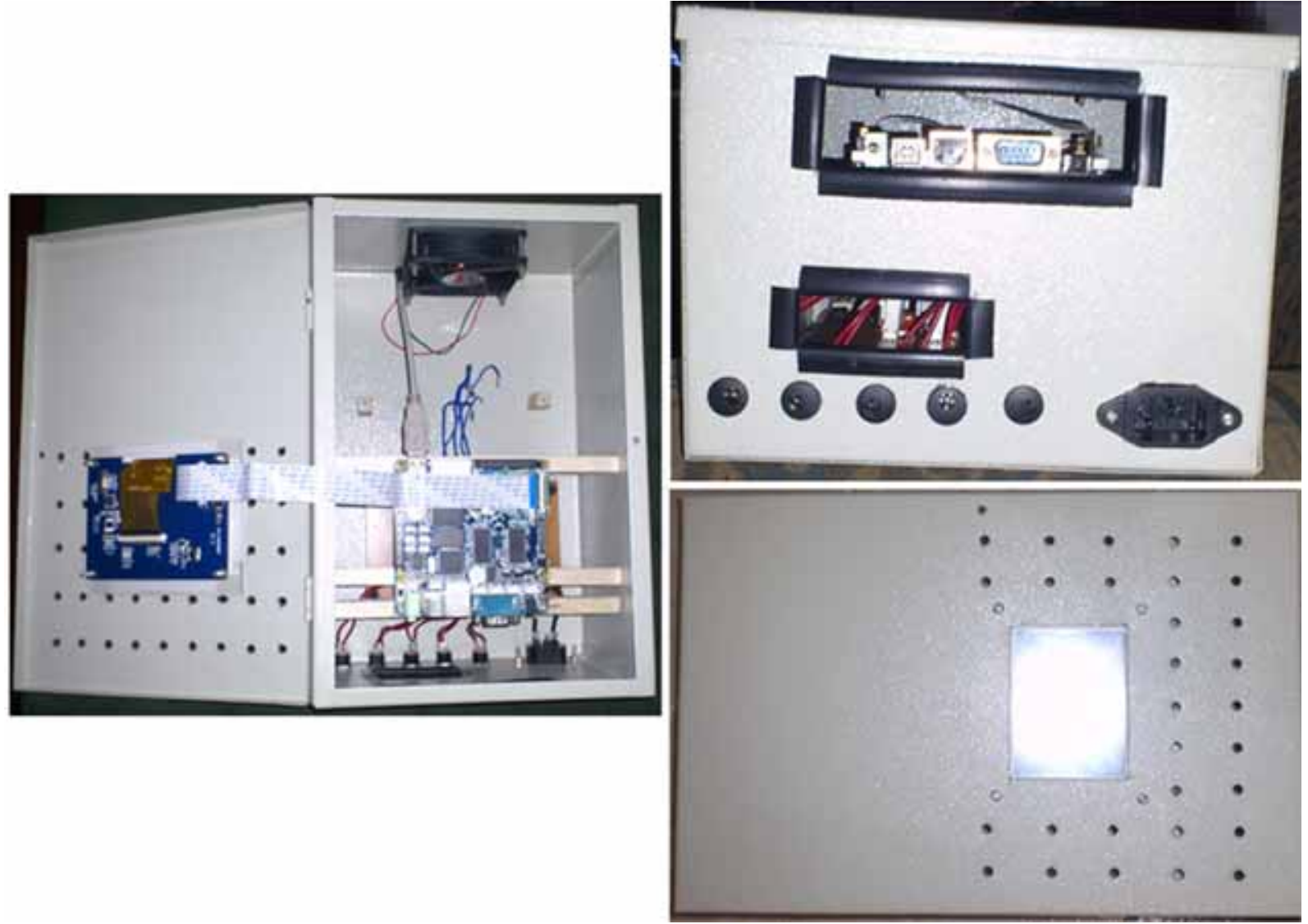

Figura 10. Caja metálica para la board ARM mini2440 y la etapa de potencia. 

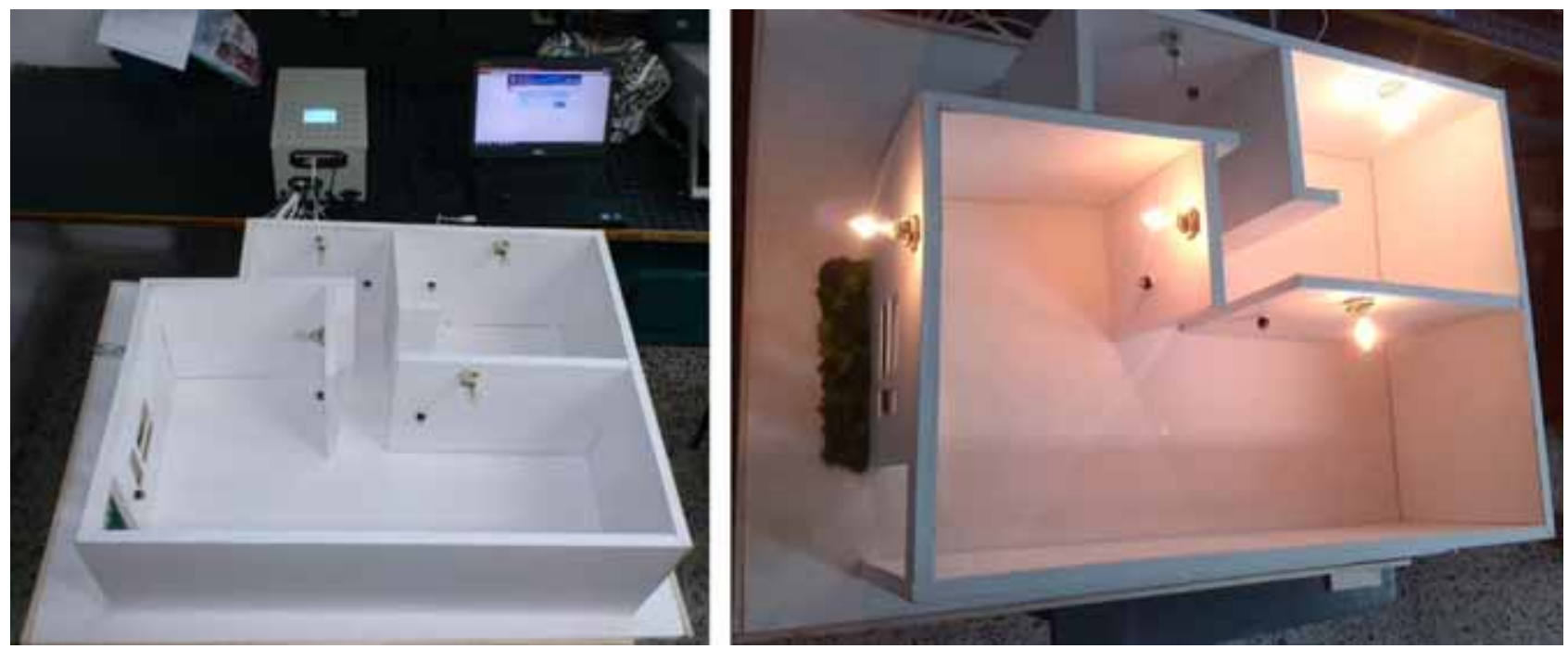

Figura 11. Acople de todo el sistema.

potencia. Esto con el fin de facilitar las conexiones, tanto de los bombillos como entre la etapa de potencia y la tarjeta. Esta caja metálica tiene unas medidas de $15 \times 15 \times 10 \mathrm{~cm}$. Se adecuó un ventilador de $12 \mathrm{~V}$ el cual va conectado directamente a la board a través de un cable USB. En la parte de arriba se encuentra la pantalla táctil de la board, la cual es protegida por un acrílico transparente, esto con el fin de que el usuario o cualquier otra persona no pueda manipularla directamente. En el frente de la caja se encuentran los conectores, tanto de los bombillos como el de la corriente para $110 \mathrm{~V}$. Dado el costo elevado para el acople de la tarjeta con una casa de verdad, se decidió construir una casa a escala (1:10) la cual trabajara con la red eléctrica de 110V (ver Figura 11).

\section{Conclusiones}

Se implementó satisfactoriamente el sistema de control y monitoreo remoto de los bombillos de una casa, gracias al uso de un sistema embebido como unidad central de procesamiento. La importancia de éste radica en la gran variedad de posibilidades que se pueden realizar utilizando una tarjeta de desarrollo como esta.

La creación de aplicaciones web con lenguaje de programación HTML5 facilita el acoplamiento con cualquier dispositivo móvil, dando la posibilidad de que la aplicación pueda ser vista desde diferentes dispositivos a través de su navegador web. Además, con variar algunos parámetros en el archivo hecho en HTML5 se puede construir una aplicación capaz de soportar diferentes sistemas operativos.
La clase proporcionada por el SDK de Android denominada WebView facilita la creación de aplicaciones web a partir de una página web, dándole al usuario la posibilidad de programar en un lenguaje diferente al nativo (Java), brindando la gran ventaja de que pueda ser vista en diferentes teléfonos móviles con sistema operativo Android sin la necesidad de crear un código para cada dispositivo y habilitar, con variar algunos parámetros, el uso de JavaScript dentro de la página web.

La base de datos admitida por la tarjeta embebida es SQLite3. A pesar de que se supone que es de bajo rendimiento, sirve perfectamente para aplicaciones que no tengan mucho manejo de datos.

\section{Referencias bibliográficas}

1. Aguiar, E., 2006. Pruebas de SQLite en un Sistema Linux - Guía para iniciarse, 1-9 pp.

2. Betancourt, L., Gómez, N., 2009. Implementación de un Sistema Teleoperado con Reaimentación Visual para Evasión de Obstáculos de un Robot Móvil.

3. Mateu, C., 2004. Desarrollo de Aplicaciones Web. Barcelona, 212-316 pp.

4. Zablaza, F., 2011. Representación de Variables Eléctricas en el Sistema Embebido mini2440. Pamplona, 9-10 pp.

5. Donelson, J., 2008. Building and Installing Software for the mini 2440, $20 \mathrm{pp}$. 
6. Herrera, L., Sánchez, S., 2012. Análisis, Diseño e Implementación de una Tarjeta de Desarrollo Utilizando el Módulo Mini2440 Basado en un Micro-Controlador ARM9 para el Laboratorio en la Carrera de Inegniería Electrónica de la Universidad Politécnica Salesiana.

7. Industrial ARMWorks, 2009. Mini2440 Hardware Essentials. Estados Unidos, 1-20 pp.

8. Finkenzeller, K. “RFID Handbook: Fundamentals and Applications in Contactless Smart Cards and Identification”. John Wiley \& Sons (May, 2012).

9. Hozak, K, Collier, David A, "RFID as an Enabler of Improve Manufacturing Performance," Decision Sciences, Volume 39 Number 4 November 2008.
10.ISO. "ISO/IEC 18000-6: Information technology automatic identification and Data capture techniques -Identificación por radiofrecuencia (RFID) a nivel de artículo- Parte 6: Parámetros de comunicación del interfaz aire a 860-960 MHz”.

11. Casadomo, 2003. El Portal de la Domótica y el Hogar Digital. Consultado el 11 de Septiembre de 2013. http://www.casadomo.com.

12.Llinares, A., 2013. Sistemas Embebidos. Consultado el 12 de Febrero de 2014. http://server-die.alc.upv. es/asignaturas/PAEEES/2005-06/A07\%20-\%20 Sistemas\%20Embebidos.pdf, 5pp. 